\title{
Palliative Toilet Mastectomy for Advanced Breast Cancer in a University Hospital of Nepal
}

\author{
Rupesh Verma, Suzita Hirachan, Yogendra P Singh \\ Breast Unit, Department of Surgery, Maharajgunj Medical Campus, Tribhuvan University Teaching Hospital, \\ Institute of Medicine, Kathmandu, Nepal
}

\section{Corresponding author:}

\section{Rupesh Verma, MBBS}

Breast Unit, Department of Surgery, Maharajgunj Medical Campus, Tribhuvan University Teaching Hospital, Institute of Medicine, Kathmandu, Nepal

Email: rups.getz@gmail.com

\begin{abstract}
\section{Introduction}

In Nepal, half of the breast cancer patients presented in advanced stage III (IIIA 18\%, IIIB 22\%), and stage IV (10\%). Delayed presentations are due to lack of awareness, reluctancy and poor accessibility to health care services often leads to local complications like sloughing of fungating breast lesions, secondary infection and bleeding. The aim of this study was to analyze the advanced breast cancer (ABC) patients who underwent palliative toilet mastectomy.
\end{abstract}

\section{Methods}

Retrospective review of all patients presenting with $A B C$ who underwent palliative toilet mastectomy in the breast unit of Tribhuvan University Teaching Hospital Kathmandu, Nepal from January 2016 to December 2018, was done. The data included were demographic profile, histopathological and immunohistochemistry report, indication of surgery, adjuvant therapy, timing of surgery, and quality of life after surgery.

\section{Results}

Total number of breast carcinoma patients was 155 within the duration of 3 years. There were 7 (4.5\%) cases of breast cancer treated with palliative toilet mastectomy with mean age of $57.9 \pm 12.7$ years. The mean tumor size was $4.3 \pm 1.3 \mathrm{~cm}$. The indications of palliative mastectomy were fungation-2 (28.5\%), bleeding-2 (28.5\%), ulceration-1 (14.3\%), malodorous discharge with secondary infection-1 (14.3\%) and hemiplegic patient with infection in $1(14.3 \%)$. Luminal A molecular subtype was seen in 1 out of 7 patients (14.3\%) with basal like subtype in 6 out of 7 patients (85.7\%). Lympho-vascular invasion were seen in 4 out of 7 (57.14\%). One patient underwent bilateral palliative mastectomy for progressive disease and margins were positive for tumor and remaining six patients had tumor free margins.

\section{Conclusion}

Advanced breast cancers are treated with neoadjuvant therapy prior surgery but the delayed presentation of these with fungating, ulcerative, infective and bleeding lesions make the initial palliative toilet mastectomy a useful option.

Keywords: Advanced breast cancer, palliative toilet mastectomy, quality of life 